\title{
THE SINGLE SERVER QUEUEING SYSTEM WITH NON-REGURRENT INPUT-PROCESS AND ERLANG SERVIGE TIME
}

\author{
P. D. FINCH
}

(received 13 September 1961)

\section{Introduction}

We consider a single server queueing system in which customers arrive at the instants $t_{0}, t_{1}, \cdots, t_{m}, \cdots$. We write $\tau_{m}=t_{m+1}-t_{m}, m \geqq 0$. There is a single server with distribution of service times $B(x)$ given by

$$
d B(x)=\mu^{k} x^{k-1} e^{-\mu x} d x /(k-1) !,
$$

where $k$ is an integer not less than unity.

We suppose that the sequence of service times $\left\{s_{j}\right\}$ is independent of the times at which customers arrive, that the $s_{j}$ are independently and identically distributed with common distribution function $B(x)$, given by (1.1), that customers are served in the order of their arrival and that a customer who arrives to find the server idle commences service immediately. We make no special assumptions about the input process $\left\{t_{n}\right\}$. Let $P_{j}^{m}\left(t_{0}, t_{1}, \cdots, t_{m}\right)$ be the conditional probability, given that the first $(m+1)$ arrivals occur at $t_{0}, t_{1}, \cdots, t_{m}$, that the arrival at $t_{m}$ finds $j$ customers in the system. We obtain explicit expressions for the probabilities $P_{j}^{m}\left(t_{0}, t_{1}, \cdots, t_{m}\right)$ and related probabilities as functions of $t_{0}, t_{1}, \cdots, t_{m}$. If the input process $\left\{t_{m}\right\}$ is a stochastic process and if $F_{m}\left(t_{0}, t_{1}, \cdots, t_{m}\right)$ is the joint distribution function of the instants at which the first $(m+1)$ arrivals occur then

$$
P_{j}^{m}=\int P_{j}^{m}\left(t_{0}, t_{1}, \cdots, t_{m}\right) d F_{m}\left(t_{0}, t_{1}, \cdots, t_{m}\right)
$$

is the probability that the $(m+1)$ th arrival finds $j$ customers in the system.

The ideas underlying the method of this paper are very simple and in order not to obscure this simplicity with algebraic manipulation we deal first with the case when the service time is exponential, that is $k=1$ in (1.1) and

$$
B(x)=1-\underset{220}{e^{-\mu x}}, \quad x \geqq 0 .
$$


Thus up to section 5 we suppose that the service time distribution is given by (1.3). In sections 6 and 7 we generalise our results to the case of the Erlang Service time distribution (1.1).

\section{Transient probabilities starting from emptiness}

Since the service time distribution is exponential and since departures can occur only when customers are in the system the instants at which customers depart form a subsequence of a sequence of potential departure points which is a Poisson process with the parameter of the exponential distribution of service time. Let $n_{m}$ be the number of potential departure points in the time interval $\left[t_{m}, t_{m+1}\right)$ of duration $\tau_{m}$. Let $\eta_{m}$ be the number of customers at the instant $t_{m}-0$. Then

$$
\eta_{m+1}=\operatorname{Max}\left[0, \eta_{m}+1-n_{m}\right] \text {. }
$$

In this section we shall suppose for simplicity that $\eta_{0}=0$, then from $(2.1)$ we obtain

$$
\begin{aligned}
\eta_{m+1}=\operatorname{Max}\left[0,1-n_{m}, 2-n_{m}-n_{m-1}, \cdots,\right. \\
\left.m+1-n_{m}-n_{m-1}-\cdots-n_{0}\right] .
\end{aligned}
$$

Now $\eta_{m+1}=j$ if and only if $k \geqq m+1-j$ potential departure points occur in $\left[t_{0}, t_{m+1}\right)$ and $\operatorname{Max}\left[0,1-n_{m}, 2-n_{m}-n_{m-1}, \cdots, m+1-n_{m}-\right.$ $\left.n_{m-1}-\cdots-n_{0}\right]=j$ with $n_{0}+n_{1}+\cdots+n_{m}=k$. But the distribution of the number of potential departure points in $\left[t_{0}, t_{m+1}\right)$ is Poisson with parameter $\mu\left(\tau_{0}+\tau_{1}+\cdots+\tau_{m}\right)$ and conditional upon there being $k$ potential departure points the instants at which they occur are independently and uniformly distributed in $\left(t_{0}, t_{m+1}\right)$. Thus if $P_{j}^{m+1}\left(\tau_{0}, \tau_{1}, \cdots, \tau_{m}\right)$ is the conditional probability that $\eta_{m+1}=j$ given $\tau_{0}, \tau_{1}, \cdots, \tau_{m}$ then

$$
P_{j}^{m+1}\left(\tau_{0}, \tau_{1}, \cdots, \tau_{m}\right)=\sum_{k=m+1}^{\infty} \mu^{k} e^{-\mu\left(\tau_{0}+\tau_{1}+\cdots+\tau_{m}\right)} \sum \frac{\tau_{0}^{n_{0}}}{n_{0} !} \frac{\tau_{1}^{n_{1}}}{n_{1} !} \cdots \frac{\tau_{m}^{n_{m}}}{n_{m} !}
$$

The second summation in (2.3) is over all non-negative integers $n_{0}, n_{1}, \cdots$, $n_{m}$ such that $n_{0}+n_{1}+\cdots+n_{m}=k$ and $\operatorname{Max}\left[0,1-n_{m}, 2-n_{m}-n_{m-1}\right.$, $\left.\cdots, m+1-n_{m}-n_{m-1}-\cdots-n_{0}\right]=j$. Equation $(2.3)$ gives $P_{j}^{m+1}$ $\left(\tau_{0}, \tau_{1}, \cdots, \tau_{m}\right)$ explicitly in terms of the inter-arrival times $\tau_{0}, \tau_{1}, \cdots, \tau_{m}$. It is possible, however, to obtain computationally simpler formulae which do not involve an infinite series. To do so we proceed as follows. First put $\mu=1$ which is no loss of generality and secondly note that if $n_{0}+n_{1}+\cdots+n_{m}<m+1-i$ then $\eta_{m+1}>i$. Then from (2.3) we have

$$
\sum_{i=0}^{j} P_{i}^{m+1}\left(\tau_{0}, \tau_{1}, \cdots, \tau_{m}\right)=e^{-\left(\tau_{0}+\tau_{1}+\cdots+\tau_{m}\right)} \sum_{k=m+1-j}^{\infty} \sum \frac{\tau_{0}^{n_{0}}}{n_{0} !} \frac{\tau_{1}^{n_{1}}}{n_{1} !} \cdots \frac{\tau_{m}^{n_{m}}}{n_{m} !} .
$$


The second summation in (2.4) is over all non-negative integers $n_{0}, n_{1}, \cdots$, $n_{m}$ such that $n_{0}+n_{1}+\cdots+n_{m}=k$ and

$\operatorname{Max}\left[0,1-n_{m}, 2-n_{m}-n_{m-1}, \cdots, m+1-n_{m}-n_{m-1}-\cdots-n_{0}\right] \leqq j$.

Observe that the inequality

$\operatorname{Max}\left[0,1-n_{m}, 2-n_{m}-n_{m-1}, \cdots, m+1-n_{m}-n_{m-1}-\cdots-n_{0}\right] \leqq j$, $0 \leqq j \leqq m$

is equivalent to the set of inequalities

$$
\begin{cases}n_{m}+n_{m-1}+\cdots+n_{m-i} \geqq 0, & 0 \leqq i<j . \\ n_{m}+n_{m-1}+\cdots+n_{m-i} \geqq i+1-j, & j \leqq i \leqq m .\end{cases}
$$

Thus using the argument which led to (2.3) but measuring the instants of potential departure from $t_{m+1}$ instead of from $t_{0}$ and taking (2.5) into account we have

where

$$
\begin{array}{r}
\sum_{i=0}^{j} P_{i}^{m+1}\left(\tau_{0}, \tau_{1}, \cdots, \tau_{m}\right)=e^{-\theta_{m, m}} \int_{0}^{\theta_{m, s}} d x_{1} \int_{x_{1}}^{\theta_{m, s+1}} d x_{2} \cdots \int_{x_{m-j-1}}^{\theta_{m, m-1}} d x_{m-j} \\
\cdot \sum_{k=m+1-j}^{\infty} \int_{x_{m-s}}^{\theta_{m, m}} d x_{m-j+1} \int_{x_{m-s+1}}^{\theta_{m, m}} d x_{m-\xi+2} \cdots \int_{x_{k-1}}^{\theta_{m, m}} d x_{k}
\end{array}
$$

$$
\theta_{m, i}=\tau_{m}+\tau_{m-1}+\cdots+\tau_{m-j}, \quad 0 \leqq j \leqq m .
$$

Noting that

$\int_{x_{m-j}}^{\theta_{m, m}} d x_{m-j+1} \int_{x_{m-j+1}}^{\theta_{m, m}} d x_{m-j+2} \cdots \int_{x_{k-1}}^{\theta_{m, m}} d x_{k}=\left(\theta_{m, m}-x_{m-j}\right)^{k-m+j} /(k-m+j) !$, we have

$$
\sum_{i=0}^{j} P_{i}^{m+1}\left(\tau_{0}, \tau_{1}, \cdots, \tau_{m}\right)=\int_{0}^{\theta_{m, s}} d x_{1} \int_{x_{1}}^{\theta_{m, s+1}} d x_{2} \cdots
$$

Define $R_{j}^{m+1}(\tau)$ by the equations

$$
\int_{x_{m-j-1}}^{\theta_{m, m-1}}\left\{e^{-x_{m-s}}-e^{-\theta_{m, m}}\right\} d x_{m-j} .
$$

$$
R_{m}^{m+1}(\tau)=e^{-\tau}-e^{-\theta_{m, m}}
$$

and

$$
\begin{array}{r}
R_{j}^{m+1}(\tau)=\int_{\tau}^{\theta_{m, 1}} d x_{1} \int_{x_{1}}^{\theta_{m, j+1}} d x_{2} \cdots \int_{x_{m-j-1}}^{\theta_{m, m-1}} R_{m}^{m+1}\left(x_{m-j}\right) d x_{m-\xi}, \\
0 \leqq j<m .
\end{array}
$$

Write

$$
R_{j}^{m+1}=R_{j}^{m+1}(0)=\sum_{i=0}^{j} P_{i}^{m+1}\left(\tau_{0}, \tau_{1}, \cdots, \tau_{m}\right)
$$


From (2.10) we find that

$$
\partial R_{j}^{m+1}(\tau) / \partial \tau=-R_{j+1}^{m+1}(\tau), \quad 0 \leqq j \leqq m
$$

with $R_{m+1}^{m+1}(\tau)=e^{-\tau}$. Thus

$$
\partial^{r} R_{j}^{m+1}(\tau) / \partial \tau= \begin{cases}(-)^{r} R_{j+r}^{m+1}(\tau) & 0 \leqq r \leqq m-j \\ (-)^{r} e^{-\tau}, & m-j+1 \leqq r\end{cases}
$$

Expanding $R_{j}^{m+1}(\tau)$ in a Taylor Series about $\tau=0$ and noting that $R_{j}^{m+1}\left(\theta_{m, j}\right)=0, j \leqq m$, we obtain

$$
0=\sum_{r=0}^{m-j}(-)^{r} \frac{\theta_{m, j}^{r}}{r !} R_{j+r}^{m+1}+\sum_{r=m-j+1}^{\infty}(-)^{r} \frac{\theta_{m, s}^{r}}{r !}, \quad j \leqq m .
$$

Writing $Q_{j}^{m+1}\left(\theta_{m, j}, \theta_{m, j+1}, \cdots, \theta_{m, m}\right)=\sum_{i=j+1}^{m+1} P_{i}^{m+1}\left(\tau_{0}, \tau_{1}, \cdots, \tau_{m}\right)$ we obtain (2.12) from(2.11)

$$
\sum_{r=0}^{m-s}(-)^{r} \frac{\theta_{m, j}^{r}}{r !} Q_{j+r}^{m+1}\left(\theta_{m, j+r}, \theta_{m, s+r+1}, \cdots, \theta_{m, m}\right)=e^{-\theta_{m, s}}
$$

Solving the system of linear equations (2.12) we obtain the following result:

THEOREM 1. If $\eta_{0}=0$ and if

$$
Q_{j}^{m+1}\left(\theta_{m, j}, \theta_{m, j+1}, \cdots, \theta_{m, m}\right)=P\left(\eta_{m+1}>j\right), \quad 0 \leqq j \leqq m
$$

where $\theta_{m, 4}$ is given by $(2.7)$ then $Q_{j}^{m+1}\left(\theta_{m, j}, \theta_{m, j+1}, \cdots, \theta_{m, m}\right)$ is given by the following determinant.

$$
(-)^{m-1}\left|\begin{array}{llllll}
d_{m, j}^{1}, & d_{m, 3}^{2}, & d_{m, j}^{3} & \cdots & d_{m, j}^{m-j} & e^{-\theta_{m, 1}} \\
1 & d_{m, j+1}^{1}, & d_{m, j+1}^{2} & \cdots & d_{m, j+1}^{m-1} & e^{-\theta_{m, s+1}} \\
0 & 1 & d_{m, j+2}^{1} & \cdots & d_{m, j+2}^{m-2,2} & e^{-\theta_{m, j+1}} \\
\cdot & \cdot & \cdot & \cdots & \cdot \\
\cdot & \cdot & \cdot & \cdots & \cdot & \cdot \\
0 & 0 & 0 & \cdots & d_{m, m-1}^{1} & e^{-\theta_{m, m-1}} \\
0 & 0 & 0 & \cdots & 1 & e^{-\theta_{m, m}}
\end{array}\right|
$$

where

$$
d_{m, j}^{r}=\left(-\theta_{m, j}\right)^{r} / r !
$$

\section{Busy period probabilities starting from emptiness}

We suppose again that $\eta_{0}=0$. We say that the busy period is still on at $t_{m+1}-0$ if $\eta_{1}>0, \eta_{2}>0, \cdots, \eta_{m+1}>0$. Let $p_{j}^{m+1}\left(\tau_{0}, \tau_{1}, \cdots, \tau_{m}\right)$ be the joint probability that the busy period is still on at $t_{m+1}-0$ and that the arrival at $t_{m+1}$ finds $j$ customers in the system. Thus 


$$
p_{j}^{m+1}\left(\tau_{0}, \tau_{1}, \cdots, \tau_{m}\right)=P\left(\eta_{1}>0, \eta_{2}>0, \cdots, \eta_{m}>0, \eta_{m+1}=j\right) .
$$

Using the notation of section 2 and on argument similar to that leading to (2.3) we see that

$$
\begin{aligned}
p_{j}^{m+1}\left(\tau_{0}, \tau_{1}, \cdots, \tau_{m}\right)=\mu^{m+1-j} e^{-\mu \theta_{m, m}} \sum \frac{\tau_{1}^{n_{1}}}{n_{1} !} \frac{\tau_{2}^{n_{2}}}{n_{2} !} \cdots \frac{\tau_{m}^{n_{m}}}{n_{m} !} \\
1 \leqq j \leqq m+1 .
\end{aligned}
$$

The summation is over all non-negative integers $n_{1}, n_{2}, \cdots, n_{m}$ such that $n_{1}+n_{2}+\cdots+n_{i} \leqq i, \quad 1 \leqq i<m$, and $n_{1}+n_{2}+\cdots+n_{m}=m-j+1$. Equation (3.2) is obtained by noting that if $\eta_{1}>0, \eta_{2}>0, \cdots, \eta_{m}>0$, $\eta_{m+1}=j$, then the total number of potential departure points (which in this case are real departure points) must be $(m+1-j)$. Conditional upon there being $(m+1-j)$ departure points the instants at which they occur are independently and uniformly distributed in $\left(t_{0}, t_{m+1}\right)$. The inequalities $n_{1}+n_{2}+\cdots+n_{i} \leqq i, 1 \leqq i<m$ specify the fact that $n_{k}>0,1 \leqq k$ $<m$.

Let $p^{m+1}\left(\tau_{0}, \tau_{1}, \cdots, \tau_{m}\right)=\sum_{j=1}^{m+1} p_{2}^{m+1}\left(\tau_{0}, \tau_{1}, \cdots, \tau_{m}\right)$ be the probability the busy period is still on at $t_{m+1}-0$. Then from (3.2) we have

$$
p^{m+1}\left(\tau_{0}, \tau_{1}, \cdots, \tau_{m}\right)=e^{-\mu \theta_{m, m}} \sum_{\substack{n_{1}+n_{2}+\cdots \\ 1 \leqq i \leqq m}} \frac{\left(\mu \tau_{1}\right)^{n_{1}}}{n_{1} !} \frac{\left(\mu \tau_{2}\right)^{n_{2}}}{n_{2} !} \cdots \frac{\left(\mu \tau_{m}\right)^{n_{m}}}{n_{m} !} .
$$

Let $l^{m+1}=P\left(\eta_{1}>0, \eta_{2}>0, \cdots, \eta_{m}>0, \eta_{m+1}=0\right)$ be the probability the arrival at $t_{m+1}$ is the first to find the server idle, that is the probability the busy period contains exactly $(m+1)$ customers. Then $l^{m+1}=p^{m}-p^{m+1}$, $p^{0}=1$ and from (3.3) we obtain

$$
\begin{aligned}
l^{m+1}=e^{-\mu \theta_{m, m}} \sum_{\substack{n_{1}+n_{2}+\cdots+n_{1} \leq l \\
n_{3}+n_{2}+\cdots+n_{m}}} \frac{\left(\mu \tau_{1}\right)^{n_{1}}}{n_{1} !} \cdots \frac{\left(\mu \tau_{m}\right)^{n_{m}}}{n_{m} !} \\
\cdot\left[1-e^{\mu \tau_{m}}\left\{1+\mu \tau_{m}+\cdots+\frac{\left(\mu \tau_{m}\right)^{n_{m}}}{n_{m} !}\right\}\right] .
\end{aligned}
$$

The results obtained above give explicit expressions for the busy period probabilities, it is interesting, however, to obtain determinantal formulae similar to those of the previous section. To do so we note that the set of inequalities

$$
n_{1}+n_{2}+\cdots+n_{i} \leqq i, \quad 1 \leqq i<m, \quad n_{1}+n_{2}+\cdots+n_{m}=m+1-j
$$

are equivalent to the following set of inequalities

$$
\begin{aligned}
& n_{m}+n_{m-1}+\cdots+n_{m+1-\xi-i} \geqq i+1, \quad 0 \leqq i<m-j \\
& n_{m}+n_{m-1}+\cdots+n_{1}=m+1-j .
\end{aligned}
$$


Thus by an argument similar to that used to derive (2.6) and putting $\mu=1$ we have

$$
p_{j}^{m+1}\left(\tau_{0}, \tau_{1}, \cdots, \tau_{m}\right)=e^{-\theta_{m, m}} \int_{0}^{\theta_{m, j-1}} d x_{m+1-j} \int_{x_{m+1-1}}^{\theta_{m, \xi}} d x_{m-j} \cdots \int_{x_{2}}^{\theta_{m, m-1}} d x_{1} .
$$

Define $q_{j}^{m+1}(\tau)$ by

$$
q_{j}^{m+1}(\tau)=\int_{\tau}^{\theta_{m, j-1}} d x_{m+1-j} \int_{x_{m+1-j}}^{\theta_{m, j}} d x_{m-j} \cdots \int_{x_{1}}^{\theta_{m, m-1}} d x_{1} .
$$

Then $p_{j}^{m+1}\left(\tau_{0}, \tau_{1}, \cdots, \tau_{m}\right)=e^{-\theta}{ }_{m, m} q_{j}^{m+1}$ where $q_{j}^{m+1}=q_{j}^{m+1}(0)$ and $q_{j}^{m+1}$ $\left(\theta_{m, j-1}\right)=0$ From (3.6) we find that

$$
\partial q_{j}^{m+1}(\tau) / \partial \tau=-q_{j+1}^{m+1}(\tau), \quad q_{m+1}^{m+1}(\tau) \equiv 1
$$

Expanding $q_{j}^{m+1}(\tau)$ in a Taylor Series about $\tau=0$ we find

$$
0=\sum_{r=0}^{m+1-j}(-)^{r} \theta_{m, j-1}^{r} q_{j+r}^{m+1} / r !
$$

with $q_{m+1}^{m+1}=1$.

Solving the set of linear equations (3.7) we obtain the following result.

THEOREM 2. If $\eta_{0}=0$ and

$$
\begin{aligned}
p_{i}^{m+1}\left(\theta_{m, j-1}, \theta_{m, 1}, \cdots, \theta_{m, m}\right)=P\left(\eta_{1}>0, \eta_{2}>0, \cdots, \eta_{m}>0, \eta_{m+1}=j\right) \\
1 \leqq j \leqq m+1
\end{aligned}
$$

where $\theta_{m, j}$ is given by (2.7) then $p_{j}^{m+1}\left(\theta_{m, j-1}, \theta_{m, j}, \cdots, \theta_{m, m}\right)$ is given by the following determinant

$$
(-)^{m+1-j}\left|\begin{array}{ccccc}
d_{m, j-1}^{1} & d_{m, 1-1}^{2} & \cdots & \cdots & d_{m, j-1}^{m+1-j} \\
1 & d_{m, j}^{1} & \cdots & \cdots & d_{m, j}^{m-j} \\
0 & 1 & \cdots & \cdots & d_{m, j+1}^{m-j-1} \\
\cdots & \cdots & \cdots & \cdots & \cdots \\
0 & 0 & \cdots & 1 & d_{m, m-1}^{1}
\end{array}\right| e^{-\theta_{m, m}}
$$

\section{Transient probabilities starting from an arbitrary state}

In this section we assume that $\mu=1$ and extend the results of section 2 to the case when the initial state is not one of emptiness. Explicitly we define

$$
P_{i, j}^{m+1}\left(\tau_{0}, \tau_{1}, \cdots, \tau_{m}\right)=P\left(\eta_{m+1}=j \mid \eta_{0}=i\right), \quad 0 \leqq i, \quad 0 \leqq j \leqq m+1+i .
$$

It is clear that if $m+1 \leqq \eta_{m+1} \leqq m+1+i$ then the busy period is still on at $t_{m+1}$ and 


$$
\begin{aligned}
P_{i, j}^{m+1}\left(\tau_{0}, \tau_{1}, \cdots, \tau_{m}\right)=\theta_{m, m}^{m+1+i-j} e^{-\theta_{m, m} /(m} & +1+i-j) ! \\
m & +1 \leqq j \leqq m+1+i .
\end{aligned}
$$

For $\eta_{m+1} \leqq m$ we argue as in section 2. Conditional upon $\eta_{0}=i$ we have

$$
\begin{array}{r}
\eta_{m+1}=\operatorname{Max}\left[0,1-n_{m}, 2-n_{m}-n_{m-1}, \cdots, m-n_{m}-n_{m-1}-\cdots-n_{1},\right. \\
\left.m+1+i-n_{m}-n_{m-1}-\cdots-n_{0}\right]
\end{array}
$$

and

$$
\sum_{n=0}^{j} P_{i, h}^{m+1}\left(\tau_{0}, \tau_{1}, \cdots, \tau_{m}\right)=e^{-\theta_{m, m}} \sum_{k=m+1+i-j}^{\infty} \sum \frac{\tau_{0}^{n_{0}}}{n_{0} !} \frac{\tau_{1}^{n_{1}}}{n_{1} !} \cdots \frac{\tau_{m}^{n_{m}}}{n_{m} !}
$$

The second summation in (4.4) is over all non-negative integers $n_{0}, n_{1}, \cdots$, $n_{m}$, such that $n_{0}+n_{1}+\cdots+n_{m}=k$ and $\eta_{m+1} \leqq j$ where $\eta_{m+1}$ is given by (4.3). As in section 2 the inequality $\eta_{m+1} \leqq j$ is equivalent to the set of inequalities

$$
\begin{array}{ll}
n_{m}+n_{m-1}+\cdots+n_{m-n} \geqq 0, & 0 \leqq h<j \\
n_{m}+n_{m-1}+\cdots+n_{m-h} \geqq h+1-j, & j \leqq h<m \\
n_{m}+n_{m-1}+\cdots+n_{0} \geqq m+1+i-j . &
\end{array}
$$

By an argument similar to that leading to (2.8) we find that

$$
\begin{aligned}
& \sum_{n=0}^{j} P_{i, h}^{m+1}\left(\tau_{0}, \tau_{1}, \cdots, \tau_{m}\right) \\
& =\int_{0}^{\theta_{m, s}} d x_{1} \int_{x_{1}}^{\theta_{m, s+1}} d x_{2} \cdots \int_{x_{m-s-1}}^{\theta_{m, m-1}}\left\{e^{-x_{m-1}}-\Gamma_{i}\left(x_{m-s}\right)\right\} d x_{m-s}
\end{aligned}
$$

where

$$
\Gamma_{i}(x)=e^{-\theta_{m, m}} \sum_{h=0}^{i}\left(\theta_{m, m}-x\right)^{n} / h !
$$

and

$$
P_{i, m}^{m+1}\left(\tau_{0}, \tau_{1}, \cdots, \tau_{m}\right)=1-\Gamma_{i}(0)
$$

Proceeding exactly as in section 2 we obtain the following result.

THEOREM 3.

Write

$$
Q_{i, j}^{m+1}=P\left(\eta_{m+1}>j \mid \eta_{0}=0\right) ; \quad 0 \leqq j \leqq m+i, \quad 0 \leqq i .
$$

Then

$$
Q_{i, j}^{m+1}=e^{-\theta_{m, m}} \sum_{n=j+1}^{m+1+i} \theta_{m, m}^{m+i-n} /(m+i-h) !, \quad m \leqq j \leqq m+i
$$

and for $0 \leqq j<m$ 


$$
Q_{i, j}^{m+1}=(-)^{m-j}\left|\begin{array}{cccccc}
d_{m, j}^{1} & d_{m, j}^{2} & d_{m, j}^{3} & \cdots & d_{m, j}^{m-j} & \phi_{m, j} \\
1 & d_{m, j+1}^{1} & d_{m, j+1}^{2} & \cdots & d_{m, j-1}^{m-j-1} & \phi_{m, j+1} \\
0 & 1 & d_{m, j+2}^{1} & \cdots & d_{m, j+2}^{m-j-2} & \phi_{m, j+2} \\
\cdots & \cdots & \cdots & \cdots & \cdots & \cdots \\
\cdots & \cdots & \cdots & \cdots & \cdots & \cdots \\
0 & 0 & 0 & \cdots & d_{m, m-1}^{1} & \phi_{m, m-1} \\
0 & 0 & 0 & \cdots & 1 & \phi_{m, m}
\end{array}\right|
$$

where

$$
\phi_{m, j}=e^{-\theta_{m, 1}}-\left[\sum_{-m-j+1}^{m-j+t}(-)^{r} \theta_{m, j}^{r} Q_{j+r}^{m+1} / r !\right], \quad 0 \leqq j \leqq m
$$

and $\theta_{m, 1}$ is given by (2.7).

\section{Busy period probabilities starting from an arbitrary state}

In this section we again assume $\mu=1$ and extend the results of section 3 to the case when the initial state is not one of emptiness. Explicitly we define

$$
\begin{array}{r}
p_{i, j}^{m+1}\left(\tau_{0}, \tau_{1}, \cdots, \tau_{m}\right)=P\left(\eta_{1}>0, \eta_{2}>0, \cdots, \eta_{m}>0, \eta_{m+1}=j \mid \eta_{0}=i\right) \\
0 \leqq i, \quad 1 \leqq j \leqq m+i+1
\end{array}
$$

Proceeding as before we find that

$$
p_{i, j}^{m+1}\left(\tau_{0}, \tau_{1}, \cdots, \tau_{m}\right)=e^{-\theta_{m}, m} \sum \frac{\tau_{0}^{n_{0}}}{n_{0} !} \frac{\tau_{1}^{n_{1}}}{n_{1} !} \cdots \frac{\tau_{m}^{n_{m}}}{n_{m} !} .
$$

The summation is over all non-negative integers $n_{0}, n_{1}, \cdots, n_{m}$ such that $n_{0}+n_{1}+\cdots+n_{m}=m+1+i-j, n_{0}+n_{1}+\cdots+n_{h} \leqq i+h, 0 \leqq$ $h<m$. The set of inequalities $n_{0}+n_{1}+\cdots+n_{h} \leqq i+h, 0 \leqq h<m$ and the equation $n_{0}+n_{1}+\cdots+n_{m}=m+1+i-j$ are equivalent to the set of inequalities $n_{m}+n_{m-1}+\cdots+n_{m-h+1} \geqq h+1-j, j \leqq h \leqq m$; $n_{m}+n_{m-1}+\cdots+n_{0}=m+1+i-j$. Hence we obtain

$$
\begin{aligned}
& p_{i, j}^{m+1}\left(\tau_{0}, \tau_{1}, \cdots, \tau_{m}\right) \\
& \quad=e^{-\theta_{m, m}} \int_{0}^{\theta_{m, s-1}} d x_{m-j+1} \int_{x_{m-j+1}}^{\theta_{m, 1}} d x_{m, j} \cdots \int_{x_{2}}^{\theta_{m, m-1}} \frac{\left(\theta_{m, m}-x_{1}\right)^{i}}{i !} d x_{1} .
\end{aligned}
$$

Proceeding as before we obtain the following result.

THEOREM 4.

$$
p_{i, j}^{m+1}=\theta_{m, m}^{m+1+i-j} e^{-\theta_{m \cdot m}} /(m+1+i-j) !, \quad m+1 \leqq j \leqq m+1+i .
$$




$$
p_{i, j}^{m+1}=(-)^{m+1-j} e^{-\theta_{m, m}}\left|\begin{array}{ccccc}
d_{m, j-1}^{1} & d_{m, j-1}^{2} & \cdots & d_{m, j-1}^{m-j+1} & \psi_{m, j-1} \\
1 & d_{m, j}^{1} & \cdots & d_{m, j}^{m-j-1} & \psi_{m, j} \\
\cdots & \cdots & \cdots & \cdots & \cdots \\
\ldots & \cdots & \cdots & \cdots & \cdots \\
0 & 0 & \cdots & 1 & \psi_{m, m-1}
\end{array}\right|
$$

where $0 \leqq j \leqq m$ and

$$
\psi_{m, j}=\sum_{r=m-j}^{m+i-j}(-)^{r} \frac{\theta_{m, j}^{r}}{r !} \frac{\theta_{m, m}^{m+i-j-r}}{(m+i-j-r) !}
$$

and $\theta_{m, 1}$ is given by (2.7).

\section{Transient probabilities for Erlang service}

In this section we suppose that the distribution of service time is given by (1.1) with $\mu=1$. We regard the arrival at $t_{m}$ as a group of $k$ individuals each having the exponential service time of (1.3) with $\mu=1$. When we wish to fix attention on the groups as single entities we refer to them as customers, if we wish to fix attention on the members of a group we talk of individuals. When the service time of an individual is given by (1.3) the distribution of the service time of a customer that is of $k$ successive individual service times is given by (1.1). Since the service time distribution of individuals is exponential and since departures of individuals from the system can occur only when individuals are in the system, the instants at which individuals depart form a subsequence of a sequence of potential departure points which form a Poisson process. Let $n_{m}$ be the number of potential departure points of individuals in the time interval $\left[t_{m}, t_{m+1}\right)$ of duration $\tau_{m}$, and let $\eta_{m}$ be the number of individuals in the system at $t_{m}-0$, then

$$
\eta_{m+1}=\operatorname{Max}\left[0, \eta_{m}+k-n_{m}\right]
$$

Write

$$
P_{i, j k+n-1}^{m+1}=P\left(\eta_{m+1}=j k+n-1 \mid \eta_{0}=i\right), \quad 0 \leqq i, \quad 0 \leqq j, \quad 1 \leqq n \leqq k
$$

then by arguments analogous to those we have used earlier we find that for $h=0,1, \cdots, i$

$$
P_{i,(m+1) k+h}^{m+1}=\left(\theta_{m, m}\right)^{i-\Lambda} e^{-\theta_{m, m}} /(i-h) !
$$

and for $0 \leqq j \leqq m, 1 \leqq n \leqq k$

$$
\sum_{n=0}^{j k+n-1} P_{i, h}^{m+1}=e^{-\theta_{m, m}} \sum_{-(m+1-j) k+i-n+1}^{\infty} \sum \frac{\tau_{0}^{n_{0}}}{n_{0} !} \frac{\tau_{1}^{n_{1}}}{n_{1} !} \cdots \frac{\tau_{m}^{n_{m}}}{n_{m} !}
$$


where the second summation is over all non-negative integers $n_{0}, n_{1}, \cdots, n_{m}$, such that $n_{0}+n_{1}+\cdots+n_{m}=r$, and $\eta_{m+1} \leqq j k+n-1$ where

$$
\begin{array}{r}
\eta_{m+1}=\operatorname{Max}\left(0, k-n_{m}, 2 k-n_{m}-n_{m-1}, \cdots, m k-n_{m}-n_{m-1}-\cdots-n_{1},\right. \\
\left.(m+1) k+i-n_{m}-n_{m-1}-\cdots-n_{0}\right) .
\end{array}
$$

It is easily verified that the inequality $\eta_{m+1} \leqq j k+n-1$ is equivalent to

$$
\left\{\begin{array}{l}
n_{m}+n_{m-1}+\cdots+n_{m-h} \geqq k(h+1-j)-n+1 ; \quad j \leqq h<m \\
n_{m}+n_{m-1}+\cdots+n_{0} \geqq k(m+1-j)+i-n+1 .
\end{array}\right.
$$

Thus we obtain $\sum_{h=0}^{j k+n-1} P_{i, h}^{m+1}=R_{i, j k+n-1}^{m+1}=R_{i, j k+n-1}^{m+1}(0)$ where

$$
\begin{aligned}
& R_{i, j k+n-1}^{m+1}(\tau)=\int_{\tau}^{\theta_{m, s}} d x_{1, n} \int_{x_{1, n}}^{\theta_{m, 3}} d x_{1, n-1} \cdots \int_{x_{1, k-1}}^{\theta_{m, 1}} d x_{1, k} \\
& \cdot \int_{x_{1, k}}^{\theta_{m, \xi+1}} d x_{2,1} \int_{x_{2,2}}^{\theta_{m, \zeta+1}} d x_{2,2} \cdots \int_{x_{2, k-1}}^{\theta_{m, 1+1}} d x_{2, k} \\
& \cdot \int_{x_{m-j-1, k}}^{\theta_{m, m-1}} d x_{m-j, 1} \int_{x_{m-j, 1}}^{\theta_{m, m-1}} d x_{m-j, 2} \cdots \\
& \text { - } \int_{x_{m-1, k-1}}^{\theta_{m, m-1}}\left\{e^{-x_{m-j, k}}-\Gamma_{k+i-1}\left(x_{m-j, k}\right)\right\} d x_{m-j, k}
\end{aligned}
$$

where $\Gamma_{i}(x)$ is given by $(4.5)$.

From (6.7) we obtain

(6.8) $\partial^{r} R_{i, j k+n-1}^{m+1}(\tau) / \partial \tau= \begin{cases}(-)^{r} R_{i, j k+n-1+r}^{m+1}(\tau), & r \leqq(m+1-j) k+i-n+1, \\ (-)^{r} e^{-\tau}, & r \geqq(m+1-j) k+i-n+1 .\end{cases}$

Expanding $R_{i, j k+n-1}^{m+1}(\tau)$ in a Taylor Series about $\tau=0$ we obtain

$$
0=\sum_{r=0}^{(m+1-j) k+i-n}(-)^{r} \frac{\theta_{m, j}^{r}}{r !} R_{i, j k+n-1+r}^{m+1}+\sum_{r=(m+1-j) k+i-n+1}^{\infty}(-)^{r} \frac{\theta_{m, j}^{r}}{r !} .
$$

\section{Writing}

$$
Q_{i, j}^{m+1}=\sum_{m=j+1}^{(m+1) k+i} P_{i, \lambda}^{m+1}
$$

we obtain for $j \leqq m, 1 \leqq n \leqq k$

$$
\sum_{r=0}^{(m-j+1) k-n}(-)^{r} \frac{\theta_{m, j}^{r}}{r !} Q_{i, j k+n-1+r}^{m+1}=e^{-\theta_{m, 1}}-\sum_{r=\langle m-j+1)^{k-n+1}}^{(m+1-j) k+i-n}(-)^{r} \frac{\theta_{m, j}^{r}}{r !} Q_{i, j k+n+r}^{m+1} .
$$

From (6.11) we can write down a determinantal expression for $Q_{i, j k+n-1+r}^{m+1}$ similar to that of Theorems 1 and 3. 


\section{Busy period probabilities for Erlang service}

In this section we make the same assumptions as in section 6. Write

$$
p_{i, j}^{m+1}=P\left(\eta_{1}>0, \eta_{2}>0, \cdots, \eta_{m}>0, \eta_{m+1}=j \mid \eta_{0}=i\right)
$$

Proceeding as before we find that

$$
p_{i, j}^{m+1}=e^{-\theta_{m}, m} \sum \frac{\tau_{0}^{n_{0}}}{n_{0} !} \frac{\tau_{1}^{n_{1}}}{n_{1} !} \cdots \frac{\tau_{m}^{n_{m}}}{n_{m} !}
$$

where the summation is over all non-negative integers $n_{0}, n_{1}, \cdots, n_{m}$ such that $n_{0}+n_{1}+\cdots+n_{h} \leqq i+(h+1) k-1,0 \leqq h<m ; n_{0}+n_{1}$ $+\cdots+n_{m}=i+(m+1) k-j$. It is easily verified that this set of inequalities is equivalent to $n_{m}+n_{m-1}+\cdots+n_{m-k+1} \geqq h k-j+1$, $1 \leqq h \leqq m, n_{m}+n_{m-1}+\cdots+n_{0}=i+(m+1) k-j$. Using the same method as before we find that for $1 \leqq j \leqq m, 1 \leqq h \leqq k$

$$
p_{i,(j-1) k+h}^{m+1}=e^{-\theta_{m, m}} q_{i,(j-1) k+h}^{m+1}(0)
$$

where

$$
\begin{aligned}
q_{i,(j-1) k+h}^{m+1}(\tau)= & \int_{\tau}^{\theta_{m, s-1}} d x_{1, n} \int_{x_{1, k}}^{\theta_{m i, j-1}} d x_{1, k+1} \cdots \int_{x_{1, k-1}}^{\theta_{m, j-1}} d x_{1, k} \\
& \cdot \int_{x_{1, k}}^{\theta_{m, 1}} d x_{2,1} \int_{x_{2,1}}^{\theta_{m, 1}} d x_{2,2} \cdots \int_{x_{2, k-1}}^{\theta_{m, 1}} d x_{2, k} \\
& \cdots \cdots \cdots \cdots \cdots \cdots \cdots \cdots \cdots \\
& \cdot \int_{x_{m-j, k}}^{\theta_{m, m-1}} d x_{m-j+1,1} \int_{x_{m-1+1,1}}^{\theta_{m, m-1}} d x_{m-j+1,2} \cdots \\
& \cdot \int_{x_{m-j+1, k-1}}^{\theta_{m, m-1}} \frac{\left(\theta_{m, m}-x_{m-j+1, k}\right)^{i+k-1}}{(i+k-1) !} d x_{m-j+1, k}
\end{aligned}
$$

for $1 \leqq j \leqq m, 1 \leqq h \leqq k$ and

$$
\begin{aligned}
p_{i, j}^{m+1}=e^{-\theta_{m, m}}\left(\theta_{m, m}\right)^{(m+1) k+i-i} /\{(m+1) k+i-j\} !, & \\
& m k<j \leqq(m+1) k+i .
\end{aligned}
$$

Proceeding as before we have

$$
\partial q_{i,(j-1) k+n}^{m+1}(\tau) / \partial \tau=-q_{i,(j-1) k+\hbar+1}^{m+1}(\tau), \quad 1 \leqq j \leqq m, \quad 1 \leqq h \leqq k .
$$

Thus we obtain

$$
\partial^{r} q_{i,(j-1) k+h}^{m+1}(\tau) / \partial^{r} \tau=(-)^{r} q_{i,(j-1) k+h+r}^{m+1}(\tau), \quad 1 \leqq j \leqq m, \quad 1 \leqq h \leqq k
$$

with the boundary condition

$$
q_{i,(m+1) k+i}^{m+1}(\tau) \equiv 1
$$


Expanding $q_{i,(j-1) k+h}^{m+1}(\tau)$ in a Taylor series about $\tau=0$ we obtain

$$
\sum_{r=0}^{(m-j+2) k+i-n}(-)^{r} \theta_{m, j-1}^{r} q_{i,(j-1) k+n+r}^{m+1} / r !=0 .
$$

From these equations we can write down a determinantal expression for the busy period probabilities as in Theorems 2 and 4 . A check can be made on equation (7.7) by putting $k=h=1$ when it becomes identical to equation (3.7).

\section{Unconditional probabilities: exponential service}

In this section we consider the case of an input process which is a stochastic process so that the random variables $\theta_{m,}$, defined by (2.7) have a joint distribution function given by

$$
F^{m+1}\left(x_{0}, x_{1}, \cdots, x_{m}\right)=P\left(\theta_{m, j} \leqq x_{j} ; 0 \leqq j \leqq m\right) .
$$

For simplicity we consider the case when the initial state is one of emptiness, and service is exponential given by (1.3) with $\mu=1$.

Write

$$
\begin{aligned}
H_{j}^{m+1}\left(\alpha_{0}, \alpha_{1}, \cdots, \alpha_{m}\right)= & E\left[Q_{j}^{m+1}\left(\theta_{m, i}, \theta_{m, j+1}, \cdots, \theta_{m, m}\right)\right. \\
& \left.\exp \left\{-\alpha_{0} \theta_{m, 0}-\alpha_{1} \theta_{m, 1}-\cdots-\alpha_{m} \theta_{m, m}\right\}\right]
\end{aligned}
$$

and

$$
\phi^{m+1}\left(\alpha_{0}, \alpha_{1}, \cdots, \alpha_{m}\right)=E\left[\exp \left\{-\alpha_{0} \theta_{m, 0}-\alpha_{1} \theta_{m, 1}-\cdots-\alpha_{m} \theta_{m, m}\right\}\right]
$$

where $\alpha_{j} \geqq 0, j \geqq 0, E[\cdot]$ denotes expectation and the $Q_{j}^{m+1}\left(\theta_{m, j}, \theta_{m, j+1}, \cdots\right.$, $\left.\theta_{m, m}\right)$ are defined in section 2. If $Q_{j}^{m+1}=E\left[Q_{j}^{m+1}\left(\theta_{m, j}, \theta_{m, j+1}, \cdots, \theta_{m, m}\right)\right]$ is the unconditional probability that $(j+1)$ or more customers are in the system on the $(m+2)$ th arrival we have

$$
Q_{j}^{m+1}=H_{j}^{m+1}(0,0, \cdots, 0)
$$

and from $(2.12)$

$$
\sum_{r=0}^{m-j} \delta_{j}^{r} H_{j+r}^{m+1}\left(\alpha_{0}, \alpha_{1}, \cdots, \alpha_{m}\right)=\phi_{j}^{m+1}\left(\alpha_{0}, \alpha_{1}, \cdots, \alpha_{m}\right)
$$

where $\delta_{j}^{r}$ denote the differential operator $(r !)^{-1} \partial^{r} / \partial \alpha_{j}^{r}$ and

$$
\phi_{j}^{m+1}\left(\alpha_{0}, \alpha_{1}, \cdots, \alpha_{m}\right)=\phi^{m+1}\left(\alpha_{0}, \alpha_{1}, \cdots, \alpha_{j-1}, \alpha_{j}+1, \alpha_{j+1}, \cdots, \alpha_{m}\right) .
$$

Solving the set equations (8.5) we obtain 


$$
H_{j}^{m+1}=(-)^{m-j}\left|\begin{array}{llllll}
\delta_{j}^{1} & \delta_{j}^{2} & \delta_{j}^{3} & \cdots & \delta_{j}^{m-j} & \phi_{j}^{m+1} \\
1 & \delta_{j+1}^{1} & \delta_{j+1}^{2} & \cdots & \delta_{j+1}^{m-j-1} & \phi_{j+1}^{m+1} \\
0 & 1 & \delta_{j+2}^{1} & \cdots & \delta_{j+2}^{m-j-2} & \phi_{j+2}^{m+1} \\
\cdot & \cdot & \cdot & \cdots & . & \cdot \\
0 & 0 & 0 & \cdots & \delta_{m-1}^{1} & \phi_{m-1}^{m+1} \\
0 & 0 & 0 & \cdots & 1 & \phi_{m}^{m}
\end{array}\right|
$$

If this determinant is evaluated at $\alpha_{0}=\alpha_{1}=\cdots=\alpha_{m}=0$ we obtain the probabilities $Q_{j}^{m+1}$. The expression (8.6) may be verified by expanding the determinant about the first row, this expansion gives equation (8.5). Denote by $\Delta_{j}^{m}$ the following determinantal differential operator, $0 \leqq j<m$.

$$
\Delta_{i}^{m}=(-)^{m-j}\left|\begin{array}{lllll}
\delta_{j}^{1} & \delta_{j}^{2} & \delta_{i}^{3} & \cdots & \delta_{j}^{m-j} \\
1 & \delta_{j+1}^{1} & \delta_{j+1}^{2} & \cdots & \delta_{j+1}^{m-j-1} \\
0 & 1 & \delta_{j+2}^{1} & \cdots & \delta_{j+2}^{m-j-2} \\
\cdot & \cdot & \cdot & \cdots & \cdot \\
\cdot & \cdot & \cdot & \cdots & \cdot \\
\cdot & \cdot & . & \cdots & \cdot \\
0 & 0 & 0 & \cdots & \delta_{m-1}^{1}
\end{array}\right|
$$

and write $\Delta_{m}^{m} \equiv 1$.

Expanding the determinant in (8.6) about its last column we obtain

$$
H_{j}^{m+1}=\sum_{s=j}^{m} \Delta_{j}^{s} \phi_{s}^{m+1} .
$$

Expanding the determinant (8.7) about its last column we obtain

$$
\Delta_{j}^{m}=-\sum_{s=j}^{m-1} \Delta_{j}^{s} \delta_{s}^{m-\varepsilon}
$$

From (8.8) we obtain

$$
Q_{j}^{m+1}=\sum_{m=j}^{m}\left[\Delta_{j}^{q} \phi_{i}^{m+1}\right]_{\alpha_{0}=\alpha_{1}=\cdots=\alpha_{m}=0} .
$$

Equations (8.10) hold for an arbitrary distribution (8.1). If the input process is stationary it is possible to simplify equation (8.10) in the following way. Suppose that the sequence of inter-arrival intervals $\left\{\tau_{m}\right\}$ is a strictly stationary stochastic process so that the joint distribution of $\left\langle\theta_{m, 0}, \theta_{m, 1}, \cdots\right.$, $\left.\theta_{m, s}\right), m>s$ is the same as the joint distribution of $\left(\theta_{s, 0}, \theta_{s, 1}, \cdots, \theta_{s, s}\right)$. From (8.3) it follows that

$$
\phi^{m+1}\left(\alpha_{0}, \alpha_{1}, \cdots, \alpha_{3}, 0,0, \cdots, 0\right)=\phi^{s+1}\left(\alpha_{0}, \alpha_{1}, \cdots, \alpha_{2}\right) .
$$


Because of (8.11) and since the operator $\Delta_{j}^{s}$ does not operate on $\alpha_{s+1}, \alpha_{s+2}$, $\cdots, \alpha_{m}$ we can write equation $(8.10)$ in the form

$$
Q_{j}^{m+1}=\sum_{s=i}^{m}\left[\Delta_{j}^{s} \phi_{s}^{s+1}\right]_{\alpha_{0}=\alpha_{1}=\cdots-\alpha_{s}=0} .
$$

We prove now

LEMma 1. If the input process is stationary and if the initial state is one of emptiness then

$$
Q_{j}=\lim _{m \rightarrow \infty} Q_{j}^{m}
$$

exists.

Proof. Observe that by (2.5) we have for $0 \leqq j<m, m \geqq 1$

$$
\begin{aligned}
1-Q_{j}^{m+1} & =P\left(n_{m}+n_{m-1}+\cdots+n_{m-i} \geqq i+1-j, i=j, j+1, \cdots, m\right) \\
& \leqq P\left(n_{m}+n_{m-1}+\cdots+n_{m-i} \geqq i+1-j, i=j, j+1, \cdots, m-1\right) \\
& \leqq P\left(n_{m-1}+n_{m-2}+\cdots+n_{m-1-i} \geqq i+1-j, i=j, j+1, \cdots, m-1\right) .
\end{aligned}
$$

The last equality being true because the input process $\left\{\tau_{m}\right\}$ is stationary. But the last expression is just $1-Q_{j}^{m}$ hence

$$
Q_{j}^{m+1} \geqq Q_{j}^{m}, \quad m \geqq 1, \quad j<m .
$$

Since $0<Q_{j}^{m}<1$ the statement of the lemma follows. Note that we have not proved the limits $Q_{j}$ form a probability distribution, we have $0<Q_{j} \leqq 1$ but the equality is not excluded.

From (8.12) and (8.13) we have

and thus

$$
\left[\Delta_{j}^{s} \phi_{s}^{s+1}\right]_{\alpha_{0}=\alpha_{1}=\cdots=\alpha_{1}} \geqq 0
$$

$$
Q_{j}=\sum_{s=j}^{\infty}\left[\Delta_{j}^{z} \phi_{s}^{o+1}\right]_{\alpha_{0}-\alpha_{1}-\cdots=\alpha_{s}=0} .
$$

The methods of this section are easily extended to the busy period probabilities, and the case of Erlang Service. No new principle is involved and we omit the details.

\section{A conjecture}

When the input process is a recurrent process, that is the inter-arrival intervals $\left\{\tau_{m}\right\}$ are independently and identically distributed with common distribution function, it is well known that

$$
Q_{3}=\left\{\begin{array}{lll}
q^{j+1} & \text { if } & \psi^{\prime}(0)+1<0 \\
1 & \text { if } & \psi^{\prime}(0)+1 \geqq 0
\end{array}\right.
$$


where $\psi(\alpha)=E \cdot e^{-\alpha r_{m}}$ and $q$ is the only (real) root within the unit circle of the equation

$$
z=\psi(1-z)
$$

when $\psi^{\prime}(0)+1<0$.

It is not easy to derive this result from the general expression (8.14). It can be shown, however, although we shall not do so here, that for a recurrent process the series (8.14) becomes

$$
Q_{j}=(j+1) \sum_{k=1}^{\infty} \frac{(-)^{k-1}}{(j+k)} \cdot \frac{1}{(k-1) !}\left[\frac{d^{k-1}}{d z^{k-1}} \psi^{j+k}(z)\right]_{z=1} .
$$

Formally this is just the series which is obtained by expanding $q^{j+1}$, where $q$ is the root of (9.2) within the unit circle, by means of Lagrange's Theorem. If $\psi^{\prime}(0)+1 \geqq 0$ there is no such root and it can be shown that the series (9.3) coverages to unity. We shall not prove these statements here because we wish to derive (9.1) by a heuristic argument. We present this heuristic discussion because it lends itself to a wider class of input process than the recurrent input process.

Write

$$
H_{j+r, j}^{m+1}\left(\alpha_{0}, \alpha_{1}, \cdots, \alpha_{j}\right)=H_{j+r}^{m+1}\left(\alpha_{0}, \alpha_{1}, \cdots, \alpha_{j}, 0,0, \cdots, 0\right) .
$$

If the input process is stationary we have from (8.10) and (8.5) that

$$
\sum_{r=0}^{m-j} \delta_{j}^{r} H_{j+r, j}^{m+1}=\phi_{j}^{j+1}
$$

Introduce a linear operator $T$, such that

$$
T_{s} \cdot H_{k, j}^{m+1}=H_{k+1, j}^{m+2}, \quad j \leqq k \leqq m .
$$

Equation (9.5) can be written in the form

$$
\sum_{r=0}^{m-j} \delta_{j}^{r} T_{j}^{+} H_{j, j}^{m+1 \rightarrow r}=\phi_{j}^{j+1} \text {. }
$$

It is easily verified that for $|z|<1$ we have

$$
\left(\sum_{k=0}^{\infty} z^{k} \delta_{j}^{k} T_{j}^{k}\right)\left(\sum_{l=0}^{\infty} z^{z} H_{j, j}^{j+l}\right)=z(1-z)^{-1} \phi_{j}^{j+1}
$$

and hence

$$
\sum_{l=1}^{\infty} z^{l} H_{j, j}^{j+l}=z(1-z)^{-1}\left(\sum_{k=0}^{\infty}(-)^{k} \delta_{j}^{k} T_{j}^{k}\right) \phi_{j}^{j+1}
$$

Equating coefficients of powers of $z$ we find 


$$
H_{j, j}^{i+k}\left(\alpha_{0}, \alpha_{1}, \cdots, \alpha_{j}\right)=\sum_{l=0}^{k-1}(-)^{l} \delta_{j}^{l} T_{j}^{l} \phi_{j}^{j+1}\left(\alpha_{0}, \alpha_{1}, \cdots, \alpha_{j}\right) .
$$

Evaluating (9.8) at $\alpha_{0}=\alpha_{1}=\cdots=\alpha_{j}=0$ we obtain the following formal expression

$$
Q_{j}^{j+k}=\sum_{l=0}^{k-1}(-)^{l}\left[\delta_{j}^{l} T_{j}^{l} \phi_{j}^{j+1}\right]_{\alpha_{0}=\alpha_{1}=\cdots=\alpha_{j}=0} .
$$

Comparing (9.9) with the previous result (8.12) we see that the correct interpretation of the terms on the right of $(9.9)$ is given by

$$
(-)^{l}\left[\delta_{j}^{l} T_{j}^{l} \phi_{j}^{j+1}\right]_{\alpha_{0}=\alpha_{1}=\cdots=\alpha_{j}=0}=\left[\Delta_{j}^{j+l} \phi_{j+l}^{j+l+1}\right]_{\alpha_{0}=\alpha_{1}=\cdots=\alpha_{j+l}=0} .
$$

With this interpretation we may proceed to the limit as in (9.9) and write

$$
Q_{j}=\sum_{l=0}^{\infty}(-)^{l}\left[\delta_{j}^{l} T_{j}^{l} \phi_{j}^{j+1}\right]_{\alpha_{0}=\alpha_{1}=\cdots-\alpha_{j}=0} .
$$

The expression on the right of (9.1) is a formal Taylor series which can be written as

$$
Q_{j}=\phi^{i+1}\left(0,0, \cdots, 0,1-T_{s}\right) .
$$

The formal expression (9.12) leads at once to a solution of the form (9.1) in the case of a recurrent input. For in that case it is easily verified that

$$
\phi^{j+1}(0,0, \cdots, 0, \alpha)=\{\psi(\alpha)\}^{j+1} .
$$

From (9.6) evaluated at $\alpha_{0}=\alpha_{1}=\cdots=\alpha_{j}=0$ we have

$$
T_{j} Q_{k}^{m+1}=Q_{k+1}^{m+2}, \quad j \leqq k \leqq m
$$

so proceeding to the limit as $m \rightarrow \infty$ we have

$$
T_{j} Q_{k}=Q_{k+1} \text {. }
$$

From (9.14), (9.13) and (9.12) we find that

$$
Q_{i}=T^{j+1}
$$

where $T=\psi(1-T)$.

Thus in a purely formal way we have deduced a solution of the form (9.1). Let us apply this formal argument to the case when the input process is a moving average of order 2 , namely

$$
\tau_{m}=u_{m+1}+\beta u_{m}, \quad m \geqq 0, \beta>0
$$

where the $u_{m}$ are non-negative, independent and identically distributed with $\psi(\alpha)=E \cdot e^{-\alpha u_{m}}$. In this case it is easily verified that

$$
\phi^{j+1}(0,0, \cdots, 0, \alpha)=\psi(\alpha)\{\psi(\overline{1+\beta} \alpha)\}^{j} \psi(\beta \alpha), \quad j \geqq 0 .
$$


The formal argument leads to a solution of the form

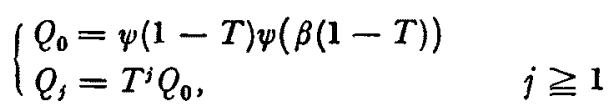

where $T$ satisfies

$$
T=\psi((1+\beta)(1-T)) .
$$

If $E\left(\tau_{m}\right)=(1+\beta) E\left(u_{m}\right) \leqq 1$ then $(9.19)$ does not possess a solution in the unit circle and we take $T=1$. The symbolic method introduced above is not intended as a proof of the result (9.18). By rather complicated more rigorous methods. I can show that when $\tau_{m}$ is given by (9.16) and $E\left(\tau_{m}\right)>1$ then $Q_{j}=T^{j} Q_{0}, j \geqq 1$ where $T$ is the root of $(9.19)$ in the unit circle but the rigorous method does not determine the constant $Q_{0}$. The symbolic method can be extended to give a solution in the case of an input process $\left\{\tau_{m}\right\}$ which is a moving average of any finite order, namely

$$
\tau_{m}=u_{m+p}+\beta_{1} u_{m+p-1}+\cdots+\beta_{p} u_{m}, \quad \beta_{j}>0, \quad 1 \leqq j \leqq p
$$

where the $\left\{u_{m}\right\}$ are non-negative identically distributed and uncorrelated. It would be interesting to see a more detailed study of the case of a moving average input process. The result (9.18) is presented here as a conjecture.

Dept. of Statistics

University of Melbourne. 